\title{
PRODUCTION OF FINE CALCIUM POWDERS BY CENTRIFUGAL ATOMIZATION WITH ROTATING QUENCH BATH
}

\author{
Liang Tian $^{\mathrm{a}, \mathrm{b}, \mathrm{c}}$, Iver Anderson ${ }^{\mathrm{a}, \mathrm{b}}$, Trevor Riedemann ${ }^{\mathrm{b}}$, Alan Russell ${ }^{\mathrm{a}, \mathrm{b}}$
}

a. Department of Materials Science and Engineering, Iowa State University, Ames, IA, 50011, USA. b. Ames Laboratory, U.S. Department of Energy, Iowa State University, Ames, IA, 50011, USA. c. Corresponding author: E-mail: lilangt@ umich.edu, Tel: +1 515509-0857, Address: 2018 Gerstacker Bldg, 2200 Bonisteel, Ann Arbor, MI, 48109, USA.

Keywords: Calcium; Centrifugal atomization; Powder; Stability; particle size; particle morphology

Abstract

Recently, a novel $\mathrm{Al} / \mathrm{Ca}$ composite was produced by severe plastic deformation of $\mathrm{Al}$ powders and $\mathrm{Ca}$ granules for possible use as a high-voltage power transmission conductor. Since the strength of such composites is inversely proportional to the Ca filament size, fine Ca powders (less than $\sim 250 \mu \mathrm{m}$ ) are needed to achieve the desired high strength for the powder metallurgy production of an Al-matrix composite reinforced by nano-scale $\mathrm{Ca}$ filaments. However, fine Ca powders are not commercially available. Therefore, we have developed a method to produce fine $\mathrm{Ca}$ powders via centrifugal atomization to supply $\mathrm{Ca}$ powder for prototype development of $\mathrm{Al} / \mathrm{Ca}$ composite conductor. A secondary goal of the project was to demonstrate that Ca powder can be safely prepared, stored, and handled and could potentially be scaled for commercial production. Our results showed that centrifugal atomization can yield as much as 83 vol. \% Ca powder particles smaller than $250 \mu \mathrm{m}$. The mean particle size sometimes matches, sometimes deviates substantially from the predictions of the Champagne \& Anger equation likely due to unexpected secondary atomization. The particle size distribution is typical for a ligament-disintegration atomization mode. Scanning 
electron micrographs showed that the morphology of these Ca powders varied with powder size. Spark testing and auto-ignition tests indicated that the atomized powders were difficult to ignite, providing confidence that this material can be handled safely in air.

\section{Introduction}

The authors are engaged in a long-term project to develop an $\mathrm{Al} / \mathrm{Ca}$ metal-metal composite (MMC) for use as a next-generation high-voltage power conductor [1]. This composite is produced by a powder metallurgy method utilizing blended $\mathrm{Al}$ and $\mathrm{Ca}$ powders. Fine $\mathrm{Al}$ powder is readily available from commercial vendors; however, fine Ca powders (a few hundred microns and smaller) are not available commercially. The strength of an $\mathrm{Al} / \mathrm{Ca}$ composite increases rapidly with decreasing $\mathrm{Ca}$ filament thickness by a Hall-Petch relation. For this reason, initial Ca powder particles with size below $250 \mu \mathrm{m}$ are necessary to achieve the desired high strength in a transmission conductor. Once achieved, the $\mathrm{Al} / \mathrm{Ca}$ composite could reduce the construction costs and increase the reliability and efficiency of high-voltage power transmission systems [2]. This paper reports on the development of a centrifugal atomization method to produce fine Ca powders that could be scaled up to meet the future needs of commercial-scale manufacturing of $\mathrm{Al} / \mathrm{Ca}$ composite conductors.

Centrifugal atomization is a well-established method for the production of fine metal powders [3-5]. This method has been successfully used to produce various metal powders such as $\mathrm{Sn}, \mathrm{Pb}, \mathrm{Al}, \mathrm{Mg}, \mathrm{Zn}, \mathrm{Ti}, \mathrm{Ni}$, and their alloys $[4,5]$. Compared with liquid or gas atomization techniques, centrifugal atomization can produce highly spherical metal powders with low impurity content, narrow particle size distributions, and high production yields [4, 5]. Centrifugal atomization with a rotating disc uses centrifugal force to disintegrate a 
molten metal stream poured directly onto the middle of a rotating disk, cup, or wheel [3] that is spinning about a vertical axis. For rotating-disk centrifugal atomization, successful atomization can be achieved only if the molten metal forms a thin liquid film on the surface of the rotating disk [3-5]. This requires excellent wettability of the molten metal on the rotating disk so the melt will flow freely on the disk's surface $[3,5]$. This liquid film will be accelerated by viscous drag between the liquid metal and rotating disk and will reach the full rotational speed by the time it experiences atomization at the disk edge [6]. These fine atomized droplets would be expected to solidify during flight by convective cooling before hitting the atomizer chamber wall or quenching medium [6].

\subsection{Atomization disk characteristics}

The design characteristics of a rotating disk atomizer have a significant effect on the performance of the atomizer [3-6]. Improper disk design can cause deviations from optimal atomization conditions, resulting in undesired powder characteristics such as large mean particle size; irregular, non-spherical particle shape; wide particle size distributions; and excessive chemical contamination [3]. An ideal atomization disk has the following characteristics: very good wetting by the liquid metal but minimal erosion/dissolution in the melt, low heat capacity and thermal conductivity, and a smooth profile that redirects the falling liquid to horizontal flow pattern [3].

The good wetting between rotating disk and molten metal ensures that the liquid would be atomized at the disk edge, as previously discussed $[3,5]$. Poor wetting (or a suboptimum melt superheat) may result in a metal skull forming on the disk, which reduces the effective radius of the atomization disk and raises the droplet size since the atomization 
occurs at the edge of the skull instead of the disk edge [7]. Moreover, the skull may detach from the atomization disk during operation, which could damage the device and degrade the atomizer performance [5]. Some investigators reported that a moderately rough surface finish on the atomization disk promoted physical bonding between the disk and skull, preventing skull detachment [3]. Wetting conditions also can be improved through design modifications of the disk. For example, a thin coating of other metals on the disk, such as Ta [3] and Sn [5], has been used to enhance wettability of a stainless steel atomization disk. Likewise, excellent wetting can be achieved between liquid metal and a coating with a composition similar to the molten metal [5]. The surface profile of an atomization disk can be designed to promote the wetting. Compared with flat disks, cup-shaped disks use centrifugal force to push the liquid metal against the disk surface to improve the wetting and to permit full liquid acceleration to be achieved [5]. In Ref [5], two methods other than promoting perfect wetting were taken to address the skull formation problem: a motor coupler was used to reduce heat conduction through the motor shaft, and a hot-air gun was used to preheat the atomization disk and maintain the disk at elevated temperature throughout the operation.

It is desirable for an atomization disk to have low heat capacity and low thermal conductivity because this allows rapid heating of the thin disk by the superheated melt, which minimizes solid metal buildup and the thick skull effect on mean particle size [3]. The break-up of the solid skull during atomization would also change the particle size distribution and powder morphology [5, 6]. Therefore, in order to eliminate fractions of irregular particles, the thickness of this solid skull layer should be minimized $[5,6]$. The chemical inertness of the atomization disk to the molten metal stream is necessary to maintain high 
purity in the metal being atomized [3]. A smooth surface profile on the atomization disk would prevent liquid splashing and direct the molten metal stream evenly to form a uniform thickness liquid metal film, narrowing the particle size distribution [3].

\subsection{Atomization modes}

Three atomization modes are typically available at the rotating disk edge as shown in Fig. 1: direct droplet formation, ligament disintegration, and film disintegration [3-6]. The transitions between these three modes depend on liquid metal flow rate, the liquid metal's physical properties, and the speed of rotation and diameter of the atomization disk [5]. Direct droplet formation is favored at low liquid flow rates and over a range of disk rotating speeds. Increasing the flow rate continuously would lead to a transition to the ligament disintegration mode first and then to film disintegration [5]. Champagne and Angers proposed an empirical formula to predict the critical flow rates of the melt corresponding to the transitions between these three modes, though the mechanisms of the transitions are not clear [8]. Considering the complexity of melt flow on an atomization disk and the speed of the disk edge, the atomization mode on the disk edge is difficult to identify as a specific mode without clear high speed image [5]. Therefore, mathematical models based on the flow behavior of liquid melt must be experimentally verified before they can be relied upon to predict particle shape and particle size distribution $[5,9]$.

Fig.1. Three atomization modes at the edge of atomization disk: direct droplet formation

(DDF); ligament disintegration (LD); film disintegration (FD) [3]. 
Ideally, centrifugally atomized powders have an approximately spherical shape. However, irregular particles have been observed frequently in centrifugally atomized $\mathrm{Al}$ and Mg alloy powders [10,11]. Various factors cause these irregular morphologies. First, during flight of liquid metal droplets, the cooling rate of fine droplets can be sufficiently fast to prevent complete spheroidization before solidification [6]. Besides, the liquid metal film may extend beyond the atomization disk edge to allow more time for cooling and delay the spheroidization. The spheroidization of these droplets is caused by the intrinsic interface perturbation coming from stagnant gas and liquid interaction according to Lord Rayleigh's instability theory [12]. The growing of the most favorable single harmonic perturbation is a kinetic process [13]. It takes more time for large droplets to complete spheroidization than it does for smaller droplets [13]. Thus, large droplets tend to develop irregular shapes, while small droplets are spherical, which has been experimentally observed by Xie et al [5].

Second, if the atmosphere in the atomizer chamber contains oxygen, rapid oxidation of the liquid metal would form stiff oxide coatings around droplets, preventing their spheroidization and further disintegration $[3,5,6]$. This leads to irregular particle morphologies and distorted particle size distributions. An inert gas atmosphere design has been adopted to produce spherical powders of oxide-forming metals with less chemical contamination [3].

Third, irregular powder morphologies can also be caused by secondary atomization via impingement on the chamber wall $[5,6]$. Small droplets cool faster due to their higher surface-to-volume ratio and fully solidify before hitting the chamber wall, so their spherical shape is maintained. Large liquid droplets have relatively slower cooling rates than small liquid droplets and they could remain partially liquid and break up into flake or splat shapes 
upon striking the chamber wall, which will also alter the particle size distribution [5]. Secondary atomization in larger droplets can be reduced by use of a high thermal conductivity gas as chamber atmosphere (e.g., He) to achieve a higher convective cooling rate [6].

In this paper, the preliminary design of a centrifugal atomizer with rotating quench bath (CARQB) has been completed for producing fine Ca powders. Funke and Anderson originally developed and built a system at Ames Laboratory to produce spherical metal powders such as Ag [7]. This design later was adapted by Osborne and Anderson to produce powders of oxidation-prone metals, such as rare earths [3]. These adaptations included using an inert gas atmosphere to replace ambient air, a Ta coated cup-shaped disk to replace a flat disk, and a more complex rotating quench bath design for particle collection and removal. This was later further modified to utilize a spray-quenching and liquid-borne collection system [3].

In our current atomizer design, low carbon steel thin sheet supported with thick ceramic thermal insulation substrate is used as the atomization disk assembly so that they can be easily heated up to be a hot disk to reduce solid Ca build-up on the atomization disk that could otherwise cause deviation from optimal operating atomization condition. The atomization disk assembly has a cup shape with a rounded center tip to direct Ca liquid stream to flow smoothly and promote the wetting between liquid $\mathrm{Ca}$ and the disk. The mass flow rate of liquid $\mathrm{Ca}$ is controlled by the Ar head pressure in the crucible to enable a stable, continuous liquid stream. The atomizer chamber is vacuumed and backfilled with ultrahigh purity inert gas (such as Ar and He) under a slight positive overpressure to prevent air and moisture contamination. The new design features of our CARQB for Ca powder production 
will be introduced in the next section. The performance of our atomizer will be evaluated by the production yield, the mean particle size, the particle size distribution, and the morphology of the Ca powders obtained.

\section{Experimental Methods}

The CARQB used to produce fine Ca powders (Fig. 2) is similar to the Ames Laboratory centrifugal atomizer with rotating quench bath developed in 1993 by Osborne and Anderson [3]. The atomization assembly (i.e. the melt supply system, atomization disk, and rotating quench bath) is housed in a mechanically pumped vacuum chamber, allowing backfilling of ultrahigh purity inert gas operated under a static positive pressure $(\leqslant 34.5$ $\mathrm{kPa}$ ). An externally mounted router motor is magnetically coupled to the atomization disk through the chamber floor, providing a rotational speed up to 24,000 RPM. A rotation speed of 20,000 RPM is chosen to produce Ca powders with size ranging from 100 to $200 \mu \mathrm{m}$ according to the empirical formula proposed by Champagne and Angers [14]. Likewise, the quench bath was driven externally at less than $200 \mathrm{rpm}$ by a motor (AMETEK Series MA34000) with integrated motor-drive controller, where coupling was via a ferrofluidic sealed feed-through.

Fig. 2. Ames centrifugal atomizer for producing fine $\mathrm{Ca}$ metal powders: mechanical design drawing (top left), photograph of the atomizer showing induction heating coil, atomization disk, and rotating quench bath (top right). A large version of mechanical design drawing is given at bottom.

The melt supply system for $\mathrm{Ca}$ atomization is comprised of a stainless steel crucible (Fig. 3) and induction heating system energized by a $9 \mathrm{KHz}, 30 \mathrm{KW}$ power supply. In our 
crucible design, a thermocouple probe (type $\mathrm{K}$ ) was immersed in the molten $\mathrm{Ca}$ to monitor the melting and superheat of the liquid metal. An over-pressure (34.5-137.9 kPa) of inert (Ar) gas was used to eject a controlled liquid $\mathrm{Ca}$ stream through the bottom orifice when the desired superheat was achieved. This method was developed to overcome two problems: (1) the low metallo-static head pressure of liquid Ca with a density of only $1.378 \mathrm{~g} / \mathrm{cm}^{3}(\sim 1 / 5$ that of $\mathrm{Sn}$ ) (a typical height of molten $\mathrm{Ca}$ is around $8 \mathrm{~cm}$, which gives a static head pressure around $1 \mathrm{kPa}$ that is negligible compared with that of pressurized Ar. This suggests that the height of liquid $\mathrm{Ca}$ have negligible effect on the liquid flow rate, which is majorly controlled by the pressurized Ar.), and (2) the high vapor pressure of $\mathrm{Ca}$, which is $0.2 \mathrm{kPa}$ at its melting point [15]. Controlling the head pressure in the crucible allows control of the mass flow rate of the $\mathrm{Ca}$ and ensures delivery of a stable, continuous liquid stream. The closed crucible effectively eliminates vapor loss of the Ca metal, which could otherwise deposit on the chamber's interior surfaces during melting and coat the observation port, blocking the operator's view of the process. Excess head pressure due to Ca vaporization and thermal expansion of trapped gas is vented through a $0.076 \mathrm{~mm}$ hole near the top of the crucible. Using water to simulate liquid Ca flow, tests showed that $69 \mathrm{kPa}$ of pressurized argon would give a proper liquid supply rate around $10 \mathrm{~cm}^{3} / \mathrm{s}$ to ensure that the atomization can be completed in a suitable time period. Before the system was induction heated, the crucible was leveled and the bottom orifice of the crucible was manually aligned vertically with the tip of the atomization disk via a (straight, narrow) tube and inspection mirror.

Fig. 3. Mechanical drawing of the crucible assembly. Dimensions are in $\mathrm{mm}$. 
The design of the atomization disk utilized a $51 \mathrm{~mm}$ diameter by $0.5 \mathrm{~mm}$ thick cupshaped disk fabricated from a low carbon steel sheet, which is supported by a $25 \mathrm{~mm}$ thick ceramic substrate of equal diameter, machined to the same profile shape. The atomization disk helps secure the ceramic substrate as its shaft penetrates the substrate and is secured to the drive shaft by a set screw. Additionally, the substrate is supported by a cup-shaped platen that is threaded on the end of the same drive shaft. The upward angle at the disk edge is 45 degrees. The center tip of the mild steel disk was rounded to promote the free flow of liquid metal on the disk and atomization took place as the liquid was accelerated off the edge of the spinning disk.

Fisherbrand D-19 mechanical vacuum pump oil was used as the quench medium. The quench basin has sufficient volume $\left(1545 \mathrm{~cm}^{3}\right)$ of oil to produce a $25 \mathrm{~mm}$-deep bath on the side while rotating at about 150 revolutions per minute (r.p.m.). This amount of oil ensures that the heat transfer from 300 grams of liquid $\mathrm{Ca}$ does not raise the temperature of this hydrocarbon oil close to its flash point $\left(212{ }^{\circ} \mathrm{C}\right)$. The rotating quench bath provides efficient cooling of liquid droplets by spreading heat uniformly inside the liquid quenchant under rotation and it was co-rotated with the atomization disk to minimize the impact velocity of the particles as they impinge upon the quenchant surface.

The crucible was loaded with 155 grams of high-purity (99.5\%) Ca granules from Minteq (Minerals Technologies Inc.) and induction heated gradually to $940{ }^{\circ} \mathrm{C}$ in the $\mathrm{Ar}$ atmosphere. A 10-minute dwell was imposed just under the melting temperature of $\mathrm{Ca}$ $\left(842^{\circ} \mathrm{C}\right)$ to ensure that the loosely loaded pellets were heated evenly. Even with the vent hole to relieve head pressure, $\mathrm{Ca}$ occasionally dripped from the melt orifice prior to initiation of stream flow due to the unsealed opening. The rotation of the atomization disk cast off the 
solidified drips and continuous steady liquid flow was initiated at full superheat by establishing $69 \mathrm{kPa}$ pressurized Ar gas head. The atomization process was recorded by a digital camera (Canon EOS Rebel T3i). After atomization, the solidified Ca powders were collected and rinsed in a hood with hexane, where six additional hexane rinses were needed to thoroughly remove the quench oil from the Ca powder surfaces. Used hexane was decanted from the cleaning vessels, while still leaving the powders submerged. These rinsed, hexane-covered $\mathrm{Ca}$ powders were placed in the antechamber of a glove box for vacuum removal of the hexane, and the dried powder was moved under inert atmosphere into the glove box. A two-gram random powder sample was pulled from the batch to measure the particle size distribution via a laser diffraction particle size analyzer (Microtrac S3500). The remaining powders were mechanically sieved into different particle size ranges. The powders at different size ranges were collected, weighed and stored in air-tight jars in the glove box. Powder samples from four different size ranges below $250 \mu \mathrm{m}$ were sealed in airtight, argon-filled vials and transported to the scanning electron microscope for morphology observations. Another set of powder samples from the same four size ranges were sparktested in air for a preliminary investigation of their ignition characteristics and any potential danger from non-inert handling fine Ca powders.

The auto-ignition temperature of these atomized and fluid quenched Ca powders (in the size range suitable for composite production) was measured in a pre-programmed muffle furnace in air atmosphere. A mass of 0.2 grams of atomized Ca powders (Mesh -70/+80, 297/+210 $\mu \mathrm{m}$ ) was used for each test. For baseline comparison, a $0.15 \mathrm{~g}$ powder sample from sublimed high purity calcium (99.95\%), prepared by the Ames Laboratory's Materials Preparation Center (MPC), was also tested after exposure to air at room temperature and 
stable formation of a native oxide. A lower mass was used due to limited availability of small particles from this preparation process. In an Ar-filled glove box, the powder samples were loaded into a small conical high-alumina crucible $(20 \mathrm{ml}$ capacity, $33 \mathrm{~mm}$ x $45 \mathrm{~mm}$, CoorsTek AD-998) and sealed in an air-tight container for transfer into the furnace. A muffle furnace (Carbolite, RHF 15/15) was used for the tests with a $900{ }^{\circ} \mathrm{C} / \mathrm{h}$ ramp rate. The sample temperatures were measured by a Type $\mathrm{K}$ thermocouple placed such that the thermocouple was in contact with the crucible bottom in the center of the crucible and the powder sample was "heaped" around the thermocouple. Firebrick and a BN crucible holder were used to position the crucible in the center of the furnace hot zone and thermocouple data was read with a data acquisition accessory (IOtech Personal Daq/56). The same apparatus and procedure was used in all tests.

\section{Results and Discussion}

\subsection{Atomization Experiments:}

The atomization disk assembly used in this study is shown in the upper left image of Fig. 4. The atomization process was captured at a frame rate of 30 fps. Fig. 5 shows a continuous series of frames at the onset of atomization. The final frame in Fig. 5 appears to show that atomization was quickly established at the disk edge. Stream flow terminated approximately $16 \mathrm{~s}$ after its initiation. At approximately $10 \mathrm{~s}$ into the atomization, the mild steel disk structurally failed and separated from the assembly, as seen in the lower left image of Fig. 4, although the welded blunted tip and shaft were still secured. Time of failure was measured by the captured video where a sudden rise of pitch from the router motor and mechanical impact noise were evident. A skull had formed on the metallic disk prior to 
failure (lower left photo in Fig. 4). The partial skull was not uniform in thickness, and it showed evidence that the atomization occurred at the disk edge. Despite the failure of the metal disk, the atomization was completed on the ceramic disk support, which was profiled to the same shape as the metal disk. A Ca skull formed and covered the entire ceramic atomization disk as shown in the upper right image of Fig. 4. Satisfactory wetting existed between the ceramic atomization disk and the liquid $\mathrm{Ca}$ metal and that the atomization of liquid $\mathrm{Ca}$ metal occurred at the disk edge as would be expected. The blunted tip design appears to improve liquid $\mathrm{Ca}$ spreading freely on the disk surface. However, the skull was rather thick, indicating that the $\mathrm{Ca}$ melt had insufficient superheat to avoid excessive solidification on, the steel first and then the ceramic surfaces. The latter could be caused by the high heat capacity of the alumina disk, which is roughly 5 times that of a Ta disk [16]. The non-uniform skull thickness may be due to liquid splashing when (apparently excessive) flow of the molten metal stream impinged on the blunted disk tip. This liquid splashing could also have contributed to the uneven distribution of liquid $\mathrm{Ca}$ on the steel disk, which may have lead to large, unevenly distributed thermal stress on the relatively weak steel disk, causing the disk failure. In our future work, the mild steel atomization disk may be replaced by a plasma-sprayed refractory metal (e.g. Ta) coating on the ceramic disk [3]. It is anticipated that Ta coated disk would perform better because Ta has an excellent wettability with most liquid metal [3] and a low heat capacity and conductivity to reduce skull formation. Besides, preheating the atomization disk would mitigate the skull formation. Currently, preheating the disk was not included in the initial design due to the limitation in the physical space of our compact chamber. It would definitely be a worthwhile improvement we shall make in the future. 
Fig. 4. The atomization disk before the experiment (upper left). The broken mild steel disk during the atomization (bottom left). The remaining solid Ca skull covered on the ceramic disk after the experiment (upper right). The ceramic disk after the skull was removed (bottom right). Disk rotation was counterclockwise.

Prior to the disk assembly failure, atomization was occurring at the disk edge as desired; under this condition, the particle size can be predicted by mathematical models. The nature of the skull in upper right picture of Fig. 4 indicated that the atomization mode at the disk edge seemed to be ligament disintegration as evidenced by solidified ligaments at the disk edge.

Champagne and Angers developed an empirical formula to calculate the critical flow rates of liquid melt $\mathrm{Q}_{\mathrm{t}}$ in $\mathrm{m}^{3} / \mathrm{s}$ corresponding to the transitions between the three modes, direct-droplet formation, ligament disintegration, and film disintegration, of centrifugal atomization $[5,8]$ :

$$
Q_{t}=\frac{K \gamma^{0.88} D^{0.68}}{\mu^{0.17} \omega^{0.6} \rho^{0.71}}
$$

Where $\gamma$ is the surface tension of the liquid metal in $\mathrm{N} / \mathrm{m}, \mathrm{D}$ is the diameter of atomization disk in $\mathrm{m}, \mu$ is the dynamic viscosity of the liquid metal in $\mathrm{Pa} \cdot \mathrm{s}, \omega$ is the rotating speed of atomization disk in $\mathrm{rad} / \mathrm{s}, \rho$ is the density of liquid metal in $\mathrm{kg} / \mathrm{m}^{3} . \mathrm{K}$ is 0.07 for the transition from direct droplet formation to ligament disintegration and 1.33 for the transition from ligament disintegration to film disintegration. In our case, the surface tension of liquid $\mathrm{Ca}$ is $0.361 \mathrm{~N} / \mathrm{m}[17,18]$, the density of liquid $\mathrm{Ca}$ is $1330 \mathrm{~kg} / \mathrm{m}^{3}$ [18], the dynamic viscosity of liquid $\mathrm{Ca}$ is $0.001012 \mathrm{~Pa} \cdot \mathrm{s}$ [19]. Disk diameter is $0.0508 \mathrm{~m}$. Disk rotating speed is $2094.4 \mathrm{rad} / \mathrm{s}$. The calculated critical liquid flow rates according to eq. (1) are $1 \mathrm{~g} / \mathrm{s}$ for direct droplet to ligament disintegration transition, $18.9 \mathrm{~g} / \mathrm{s}$ for ligament to film 
disintegration transition. Around 134.6 grams of liquid Ca metal was ejected onto the disk during an approximate time period of $16 \mathrm{~s}$, which gives the liquid flow rate $8.4 \mathrm{~g} / \mathrm{s}$ in our experiment. This liquid flow rate is between the two critical flow rates, which means that the atomization mode at disk edge in our experiment should be ligament disintegration. The spiral ligaments around the disk edge in Fig. 5 seem to match the ligament disintegration atomization mechanism.

Fig. 5. Continuous photograph frames of the initiation of liquid Ca metal atomizing with time progressing from the top to the bottom under Ar atmosphere at $30 \mathrm{fps}$.

3.2 Particle Size Distribution and Production Yield:

Particle size distribution is the most important characteristic of a powder sample and is commonly used to evaluate the performance of a centrifugal atomizer. Particle size distribution is strongly dependent on atomization mechanisms, which are determined by the operating parameters and the physical properties of the liquid metal being atomized [8]. The particle size distributions of two, $2 \mathrm{~g}$ random clean Ca powder samples from each of the two atomization runs produced from our centrifugal atomizer are shown in Fig. 6. For the first atomization run, the mean particle sizes, $\mathrm{d}_{50}$, for these two distributions were $126.1 \mu \mathrm{m}$ and $123.8 \mu \mathrm{m}$. For the second atomization run, the $\mathrm{d}_{50}$ values were $168.2 \mu \mathrm{m}$ and $181.8 \mu \mathrm{m}$. Champagne and Angers proposed an empirical formula to predict the mean particle size $\mathrm{d}_{50}$ (meter) of centrifugally atomized powders [3, 14]:

$$
d_{50}=\frac{3.65 Q^{0.06}}{\omega D^{0.58}}\left(\frac{\gamma}{\rho}\right)^{0.46}
$$


,where $\mathrm{Q}$ is the liquid flow rate in $\mathrm{m}^{3} / \mathrm{s}$, $\omega$ is the disk rotational speed in $\mathrm{rad} / \mathrm{s}, \mathrm{D}$ is the disk diameter in $\mathrm{m}, \gamma$ is the surface tension of liquid metal in $\mathrm{N} / \mathrm{m}, \rho$ is the density of liquid metal in $\mathrm{kg} / \mathrm{m}^{3}$. In our experiment, $\mathrm{Q}=6.3 \times 10^{-6} \mathrm{~m}^{3} / \mathrm{s}, \omega=2094.4 \mathrm{rad} / \mathrm{s}, \mathrm{D}=0.0508 \mathrm{~m}, \gamma=0.361 \mathrm{~N} / \mathrm{m}$, $\rho=1330 \mathrm{~kg} / \mathrm{m}^{3}$ [17-19]. According to eq. (2), the calculated mean particle size would be $109.5 \mu \mathrm{m}$. The average of two mean particle sizes of the first atomization run is $125 \mu \mathrm{m}$, which is $14 \%$ larger than this prediction but roughly matches the predicted mean particle size. The average of two mean particle sizes of the second atomization run is $175 \mu \mathrm{m}$, which is $60 \%$ larger than the predicted mean particle size. There are several possible reasons for this deviation of mean particle size from the predicted value. According to eq. (2), the mean particle size depends on rotating speed and diameter more strongly than other parameters. A $10 \%$ reduction in rotating speed would change the mean particle size to $121.6 \mu \mathrm{m}$. Though we have calibrated the continuous dial setting of the speed controller, it is possible that combination of measurement error from the tachometer, minor dial-setting error during operation, and liquid $\mathrm{Ca}$ load-induced reduction in rotating speed would contribute to the deviation of rotating speed from 20,000 rpm. It is also possible that a small fraction of liquid $\mathrm{Ca}$ atomized from the skull instead of the disk edge, which reduced the effective atomization radius and increased the mean particle size. Moreover, the Champagne and Angers equation is an empirical equation based on regression analysis of several more conventional metals with far different physical properties, although the physics of the centrifugal process is identical. For example, the density of liquid Ca metal is only $1330 \mathrm{~kg} / \mathrm{m}^{3}$, outside of the density range $2000-8000 \mathrm{~kg} / \mathrm{m}^{3}$ in Champagne and Angers equation [14] and the surface tension of $0.361 \mathrm{~N} / \mathrm{m}$ is outside of the range $0.7-1.8 \mathrm{~N} / \mathrm{m}$ that they studied. In terms of operating parameters, the liquid flow rate $6.3 \mathrm{~cm}^{3} / \mathrm{s}$ is also outside of the range $0.06-0.8$ 
$\mathrm{cm}^{3} / \mathrm{s}$ in Champagne and Angers equation. From the literature, most experimental studies investigated the effect of each processing parameters on mean particle size $[5,6,20]$ and some studies did show a contradiction with Champagne and Angers equation, e.g. Plookphol et al [21] found a stronger dependence of the mean particle size on liquid flow rate. This being said, the most significant influence on the particle size results was probably the offnormal buildup of the solidified skull on the disk surface and the disk rupture event that occurred midway through the atomization process. Thus, our plan is to achieve more controlled disk surface conditions by a change in the disk construction (see above) and an increased superheat, both of which should permit a more critical study of the effects of operating parameters and physical properties of the liquid metal on the mean particle size in the future.

Fig. 6. The particle size distributions of 2 random, clean, atomized Ca powder samples for each of two atomization runs in Ar chamber atmosphere using the same experimental apparatus. The samples were run through a $1 \mathrm{~mm}$ screen prior to analysis.

In spite of some deviation from the desired steady state atomization mechanism, Fig. 6 shows that the majority powders sit in the size range from $60 \mu \mathrm{m}$ to $320 \mu \mathrm{m}$, which is the desired size range needed to produce test samples for the proposed $\mathrm{Al} / \mathrm{Ca}$ composite conductor. This particle size distribution would not be expected if the direct droplet formation was the primary atomization mechanism. The direct droplet formation mechanism would generate an obvious bimodal particle size distribution with the main and secondary particles forming two different modes [8]. In addition, the mode from secondary particles should have much less volume fraction than that of the mode from main particles, considering the number of secondary particles is roughly equal to that of main particles [8]. 
The particle size distribution in Fig. 6 clearly deviates from the above description, i.e the volume fraction of main particles is roughly equal to that of secondary particles. Increasing the liquid flow rate would lead to the gradual transition of atomization mode from direct droplet formation to ligament disintegration. As discussed in Section 3.1, the liquid flow rate in our experiment would be predicted by theory to operate in the liquid disintegration atomization mode. It has been observed that the proportion of secondary particles increases steadily with the liquid flow rate [8]. This can explain the fact that the volume fraction of the secondary particles is roughly equal to that of the main particles observed in Fig. 6. This feature of particle size distribution further indicated the ligament disintegration atomization mechanism was dominant in our experiment.

In addition to the atomization mode, the operating conditions and properties of the liquid metal (as mentioned above) can also affect particle size distribution by: (1) forming a thick skull that could shift the particle size to a coarser range; (2) the non-uniform skull thickness on the ceramic disk that could widen the particle size distribution. The particle morphology can further alter the measured particle size distribution as well. For example, non-spherical particles like flakes, splats, and rods could result in a bimodal particle size distribution as well, since the Microtrac unit could mistakenly read the two different dimensions of these irregular particles as two different particle sizes [2].

Dry, clean $\mathrm{Ca}$ powders produced from one run of our centrifugal atomizer were mechanically sieved into different particle size ranges and weighed, as shown in Table 1 . About half of these Ca powders ranged from $74 \mu \mathrm{m}$ to $149 \mu \mathrm{m}$, and $83 \%$ of the Ca powders have particle size less than $250 \mu \mathrm{m}$. This demonstrates that our centrifugal atomizer is capable of producing a high yield of the desired fine Ca powders. 
Table 1. The powder production yields for different particle size ranges.

\subsection{Particle Morphology:}

Particle morphology is another important powder characteristic that indicates the quality of the centrifugally atomized powders. Ideally, spherical powders are expected for centrifugally atomized powder. However, irregular powder shapes are frequently observed in centrifugal atomization for various reasons $[5,6,8,20,22]$. In our experiment, SEM micrographs of $\mathrm{Ca}$ powders with four different particle size ranges were taken to examine their morphologies. As shown in Fig. 7, large Ca powders (i.e. $177 \mu \mathrm{m} \sim 250 \mu \mathrm{m}$ ) tend to have irregular ligamental, rodlike shapes, while smaller Ca powders (i.e. $74 \mu \mathrm{m} \sim 105 \mu \mathrm{m}$ ) are predominantly spherical. As the Ca powder size decreases, a gradual transition of particle shape is observed from irregular to spherical.

Various factors can explain the change of particle morphology with particle size. First, liquid droplets atomized at the disk edge have sufficiently fast cooling rates to solidify in spherical form during flight. Larger droplets may not have sufficient time to spheroidize before solidification so that ligament, rod, and teardrop shaped powders would be produced, while small droplets completed spheroidization before solidification to develop a spherical shape. If irregular-shape $\mathrm{Ca}$ powders were produced in this way, the surface of these $\mathrm{Ca}$ powders would be smooth due to a solidification process $[6,8]$, as can be seen in the smooth solidified surface of Ca powders of 74-105 $\mu \mathrm{m}$ in Fig. 7. However, it is clear in Fig. 7 that a substantial fraction of $\mathrm{Ca}$ powders of $177-250 \mu \mathrm{m}$ have rough surfaces and tortured shapes 
other than ligament or spheroid. To explain these characteristics, we propose that spherodization was interrupted as the larger, partially liquid $\mathrm{Ca}$ droplets impinged on the quench bath. Due to a smaller surface to volume ratio, larger Ca liquid droplets have a slower cooling rate than smaller $\mathrm{Ca}$ liquid droplets so that larger $\mathrm{Ca}$ droplets may still be semi-solid and not fully solidified before hitting the rotating quench bath. The impingement of partly liquid Ca droplets upon the quench bath would substantially distort the morphology of the droplets and create rough surfaces and tortured shapes. Small, spheroidized Ca droplets would fully solidify before hitting the quench bath resulting in spherical particles with smooth surfaces.

Fig. 7. Scanning electron microscope (SEM) images of centrifugally atomized Ca powders for four different size ranges: 177-250 $\mu \mathrm{m}$ (upper left); 149-177 $\mu \mathrm{m}$ (upper right); 105-149 $\mu \mathrm{m}$ (lower left); 74-105 $\mu \mathrm{m}$ (bottom right). The chamber was filled with Ar gas.

Confirmation of this mechanism has been done by filling the chamber with He, which has a higher thermal conductivity than Ar and performing an additional atomization run under very similar conditions. Helium gas will increase the cooling rate of the liquid $\mathrm{Ca}$ droplets during flight; thus, it would be expected that a larger portion of droplets will fully solidify before reaching the quench bath, increasing the fraction of spherical particles. Fig. 8 shows the SEM pictures on powder morphology of Ca powders atomized in He. It can be clearly seen that the transition from irregular particle shape to spherical particle shape occurred at 149-177 $\mu \mathrm{m}$ for powders produced in a He atmosphere, compared with 105-149 $\mu \mathrm{m}$ for powders produced under Ar. The more rapid cooling effect of He allowed larger size powders to be fully solidified into spheres before hitting the quench bath, while the slower cooling of Ar gas can allow only smaller powders to be fully solidified and spherical. All the 
above evidence confirmed that the irregular shape is caused by a premature quench when the partially solidified larger Ca droplets hit upon the liquid quench bath.

Fig. 8. Scanning electron microscope (SEM) images of centrifugally atomized Ca powders for four different size ranges: 177-250 $\mu \mathrm{m}$ (upper left); 149-177 $\mu \mathrm{m}$ (upper right); 105-149 $\mu \mathrm{m}$ (lower left); 74-105 $\mu \mathrm{m}$ (bottom right). The chamber was filled with He gas.

It is worth noting that some fraction of these irregular powders probably come from the non-ideal break-up of the melt from the thick solidified skull at a reduced diameter, resulting in significant production of the larger size droplets than the intended size $[5,6]$. Thus, changes in the experimental conditions that favor more ideal breakup should produce smaller liquid $\mathrm{Ca}$ droplets and would increase the fraction of spherical powders.

\subsection{Surface Reactivity and Safe Handling:}

Throughout the development of CARQB, safe handling of Ca powders has been a major concern. $\mathrm{Ca}$ is a reactive metal that readily oxidizes in air and water. According to Laurendeau and Glassman, the ignition temperature for bulk calcium metal is around $790{ }^{\circ} \mathrm{C}$; for bulk $\mathrm{Mg}$ metal, it is $635^{\circ} \mathrm{C}$ [23]. Prior to any atomization attempts, spark testing of handfiled fine Ca debris showed little reactivity when exposed to ambient atmosphere conditions, and no sustained burn could be initiated with the Telsa coil. This suggested that large quantities of fine Ca powders could be handled safely [24] if not exposed to high temperature or other conditions that promoted rupture of the native oxide, which is similar to that of other metal powders already produced on a large scale such as $\mathrm{Mg}$ and $\mathrm{Al}$ powders passivated with a native oxide. 
Following centrifugal atomization and quenching in the hydrocarbon oil bath, four $\mathrm{Ca}$ powder samples from the particle size ranges shown in Fig. 7 were spark tested in air, using Tesla coil in an attempt to ignite each sample. Our results showed that powders with mesh size $+80 /-60$ had no reaction; powders with mesh size $+100 /-80$ had a slight glow that was not sustained when the spark source was removed; powders with mesh size $+150 /-100$ and $+200 /-150$ would glow but with little or no propagation when the spark source was removed. These simple tests suggest that fine Ca powders quenched in oil pose no extraordinary hazard for safe handling. The surface inertness of these fine $\mathrm{Ca}$ powders could originate from a passivation surface layer on these Ca powders due to the chemical reaction with the liquid quenchant or a partially oxidized surface.

To further verify the stability of atomized $\mathrm{Ca}$ powders, auto-ignition tests were conducted on atomized Ca powders (Mesh size -70/+80, 177-210 $\mu \mathrm{m}$ ) and Ames Lab highpurity (99.95\%) Ca powders (Mesh size $-70 /+80,177-210 \mu \mathrm{m}$ ) as a case for comparison. The tests were conducted in a furnace under room air with a $900{ }^{\circ} \mathrm{C} /$ hour ramping rate. Fig. 9 shows the ignition temperature of two types of samples varying as a function of time. The atomized Ca powders were clearly stable until $745^{\circ} \mathrm{C}$, and then the protective layer broke so that pure $\mathrm{Ca}$ was exposed to air and oxidized rapidly. In contrast, the high-purity Ca powders were stable only to $665{ }^{\circ} \mathrm{C}$. Before this temperature, they were presumably protected by a thin layer of native oxide and hydroxide [23]. While this amount of stability for the native oxide coating may seem higher than expected, Steinmetz demonstrated that the ignition temperature for a $-63 \mu \mathrm{m}$ cut of commercial $\mathrm{Mg}$ powders is $525^{\circ} \mathrm{C}$ in air [25]. Fig. 10 shows that the particle morphology of the sublimed particles (baseline) is similar to that of the atomized powders in this size range (i.e. non-spherical), such that the difference in ignition 
temperatures is likely due to the difference in surface chemistry. Therefore, our results suggested that the atomized and oil quenched Ca powders exhibit enhanced thermal stability over the baseline $\mathrm{Ca}$ powders with only a native oxide coating and pose a significantly reduced ignition hazard. It is worthwhile to discuss the effect of the protective layer on the electrical conductivity and mechanical strength of $\mathrm{Al} / \mathrm{Ca}$ composite synthesized by these $\mathrm{Ca}$ powders. Our experimental resistivity measurements suggested that the $\mathrm{Al} / \mathrm{Ca}$ (20 vol.\%) composite synthesized by these Ca powders have a resistivity $0.0305 \mu \Omega \cdot \mathrm{m}$, which is only $3.4 \%$ higher than the predicted $0.0295 \mu \Omega \cdot \mathrm{m}$ by the inverse rule of mixtures and still lower than most currently available conductors. This suggests that the protective layer has negligible effect on the resistivity of $\mathrm{Ca}$ powder and $\mathrm{Al} / \mathrm{Ca}$ composite. Furthermore, the mechanical strength of $\mathrm{Al} / \mathrm{Ca}$ composite comes from the $\mathrm{Al} / \mathrm{Ca}$ interface that act as barrier to dislocation motion to strengthen the composite, rather than the strength of Ca itself [1]. Our strength measurements of $\mathrm{Al} / \mathrm{Ca}$ composite suggested that the composite strength showed a good match with interface strengthening (Hall-Petch barrier) model to yield a very high strength $480 \mathrm{MPa}$ (considerably higher than all current conductors), which suggests that the effect of protective layer on the strength of $\mathrm{Al} / \mathrm{Ca}$ composite is negligible.

Fig. 9. Auto-ignition tests on atomized Ca powders (Mesh -70/+80) and Ames Lab highpurity Ca powder (Mesh $-70 /+80)$ in air.

Fig. 10. Scanning Electron Microscopy (SEM) image of the particle morphology of Ames Lab high-purity sublimed Ca powders (Mesh -50/+70).

\author{
4. Conclusions
}


Fine $\mathrm{Ca}$ metal powders have been produced by a rotating disk centrifugal atomizer with a rotating quench bath under an inert atmosphere. The performance of the centrifugal atomizer was evaluated by post-atomization observations, such as solid skull thickness and uniformity, and various powder characteristics such as mean particle size, particle size distribution, production yield and particle morphology. Our results support the conclusion that the atomization of liquid $\mathrm{Ca}$ metal occurred at the edge of the atomization disk by a ligament disintegration atomization mode. The production yield of desired fine Ca powders less than $250 \mu \mathrm{m}$ is as high as $83 \%$ of the total powders produced by the centrifugal atomizer. SEM micrographs showed that the larger Ca powders (i.e. 177-250 $\mu \mathrm{m}$ ) tend to have irregular shapes, and the smaller Ca powders (i.e. 74-105 $\mu \mathrm{m}$ ) tend to be spherical. This can be explained by distortion of the particle morphology when larger partially liquid Ca droplets impinged upon the quench bath. Spark testing and auto-ignition tests showed that these fine atomized Ca powders were thermally stable (more stable than high-purity Ca powders in the same size range) and thus should be safe to handle.

\section{Acknowledgements}

The authors are grateful for the financial support of ISU's Electric Power Research Center, Iowa State University Research Foundation, U.S. Department of Energy Office of Electricity, and Summit Technology Group LLC. Additionally, the authors appreciate the help of Alon Klenkner and Terry Herrman from Ames Laboratory’s Engineering Service Group (ESG) on fabrication of centrifugal atomizer apparatus. 


\section{References}

[1] L. Tian, H. Kim, I. Anderson, A. Russell, The microstructure-strength relationship in a deformation processed Al-Ca composite, Mat Sci Eng a-Struct, 570 (2013) 106-113.

[2] L. Tian, I. Anderson, T. Riedemann, A. Russell, H. Kim, Prospects for novel deformation processed $\mathrm{Al} / \mathrm{Ca}$ composite conductors for overhead high voltage direct current (HVDC) power transmission, Electr Pow Syst Res, 105 (2013) 105-114.

[3] M.G. Osborne, Centrifugal atomization of lanthanide materials for cryogenic coolers, Other Information: TH: Thesis (M.S.); PBD: 4 Jan 1994, 1994, pp. Medium: ED; Size: $106 \mathrm{p}$.

[4] T.P. Phairote Sungkhaphaitoon, and Sirikul Wisutmethangoon, Design and Development of a Centrifugal Atomizer for Producing Zinc Metal Powder, International Journal of Applied Physics and Mathematics, 2 (2012).

[5] J.W. Xie, Y.Y. Zhao, J.J. Dunkley, Effects of processing conditions on powder particle size and morphology in centrifugal atomisation of tin, Powder Metall, 47 (2004) 168-172.

[6] R. Angers, R. Tremblay, D. Dube, Formation of irregular particles during centrifugal atomization of AZ91 alloy, Mater Lett, 33 (1997) 13-18.

[7] K. Funke, Development of a centrifugal atomization process for the production of large spherical metal powders, Ames Lab., IA (United States), 1991.

[8] B. Champagne, R. Angers, Rep Atomization Mechanisms, Powder Metall Int, 16 (1984) 125-128.

[9] Y.Y. Zhao, M.H. Jacobs, A.L. Dowson, Liquid flow on a rotating disk prior to centrifugal atomization and spray deposition, Metall Mater Trans B, 29 (1998) 1357-1369.

[10] R. Angers, C. Dube, R. Tremblay, Inverted disk centrifugal atomization of 2024, Int J Powder Metall, 30 (1994) 429-434.

[11] C. Labrecque, R. Angers, R. Tremblay, D. Dube, Inverted disk centrifugal atomization of AZ91 magnesium alloy, Canadian metallurgical quarterly, 36 (1997) 169-175.

[12] J.W. Strutt, L. Rayleigh, On the instability of jets, Proc. R. Soc. London A, 1879, pp. 413.

[13] L. Tian, A. Russell, Phase field study of interfacial diffusion-driven spheroidization in a composite comprised of two mutually insoluble phases, The Journal of Chemical Physics, 140 (2014). 
[14] B. Champagne, R. Angers, Size distribution of powders atomized by the rotating electrode process, Modern Development in Powder Metallurgy, 12 (1980) 83-104.

[15] R.E. Honig, Vapor Pressure Data for the Solid and Liquid Elements, Rca Rev, 23 (1962) 567-586.

[16] http://www.matweb.com/, May 19, 2014.

[17] B.J. Keene, Review of Data for the Surface-Tension of Pure Metals, Int Mater Rev, 38 (1993) 157-192.

[18] Bohdansk.J, H.E.J. Schins, Surface Tension and Density of Liquid Earth Alkaline Metals Mg Ca Sr Ba, J Inorg Nucl Chem, 30 (1968) 2331-\&.

[19] M.F. Culpin, The Viscosity of Liquid Magnesium and Liquid Calcium, P Phys Soc Lond B, 70 (1957) 1079-1086.

[20] S. Ozturk, F. Arslan, B. Ozturk, Effect of process parameters on production of metal powders by water jet cooled rotating disc atomisation, Powder Metall, 48 (2005) 163170.

[21] T. Plookphol, S. Wisutmethangoon, S. Gonsrang, Influence of process parameters on SAC305 lead-free solder powder produced by centrifugal atomization, Powder Technology, 214 (2011) 506-512.

[22] P. Sungkhaphaitoon, W. Likhidkan, S. Kitjaidiaw, S. Wisutmethangoon, T. Plookphol, Effect of Atomizer Disc Geometry on Zinc Metal Powder Production by Centrifugal Atomization, Appl Mech Mater, 271-272 (2013) 232-236.

[23] N.M. Laurendeau, I. Glassman, Ignition Temperatures of Metals in Oxygen Atmospheres, Combustion Science and Technology, 3 (1971) 77-82.

[24] I.E. Anderson, B.K. Lograsso, R.L. Terpstra, Environmentally stable reactive alloy powders and method of making same, US5811187 A, (1998).

[25] A.D.Steinmetz, Investigation of a novel passivation technique for gas atomized magnesium powders, Iowa State University thesis, 2011. 
Table 1. The powder production yields for different particle size ranges.

\begin{tabular}{|l|l|l|l|}
\hline $\begin{array}{l}\text { U.S. mesh } \\
\text { size }\end{array}$ & Powder size & Powder weight $(\mathrm{g})$ & $\begin{array}{l}\text { Percentage of } \\
\text { total mass } \\
\text { collected }\end{array}$ \\
\hline+18 & $>1 \mathrm{~mm}$ & 8.920 & $8.7 \%$ \\
\hline$+30 /-18$ & $595 \mu \mathrm{m}-1 \mathrm{~mm}$ & 1.562 & $1.5 \%$ \\
\hline$+60 /-30$ & $250-595 \mu \mathrm{m}$ & 6.794 & $6.6 \%$ \\
\hline$+80 /-60$ & $177-250 \mu \mathrm{m}$ & 11.045 & $10.7 \%$ \\
\hline$+100 /-80$ & $149-177 \mu \mathrm{m}$ & 10.622 & $10.3 \%$ \\
\hline$+150 /-100$ & $105-149 \mu \mathrm{m}$ & 23.820 & $23.1 \%$ \\
\hline$+200 /-150$ & $74-105 \mu \mathrm{m}$ & 26.819 & $26.0 \%$ \\
\hline-200 & $<74 \mu \mathrm{m}$ & 13.491 & $13.1 \%$ \\
\hline & & Total: 103.073 & \\
\hline
\end{tabular}




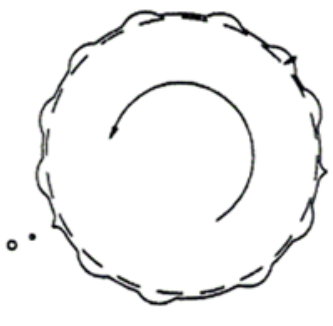

DDF

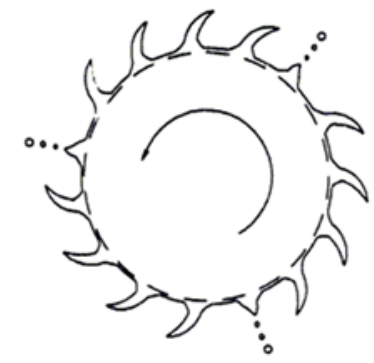

LD

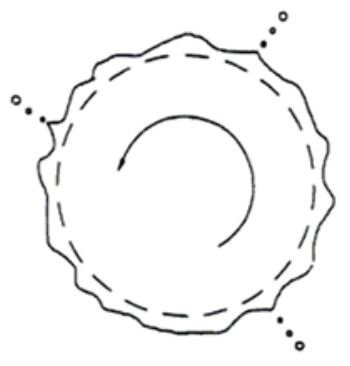

FD

Fig.1. Three atomization modes at the edge of atomization disk: direct droplet formation (DDF); ligament disintegration (LD); film disintegration (FD) [3]. 


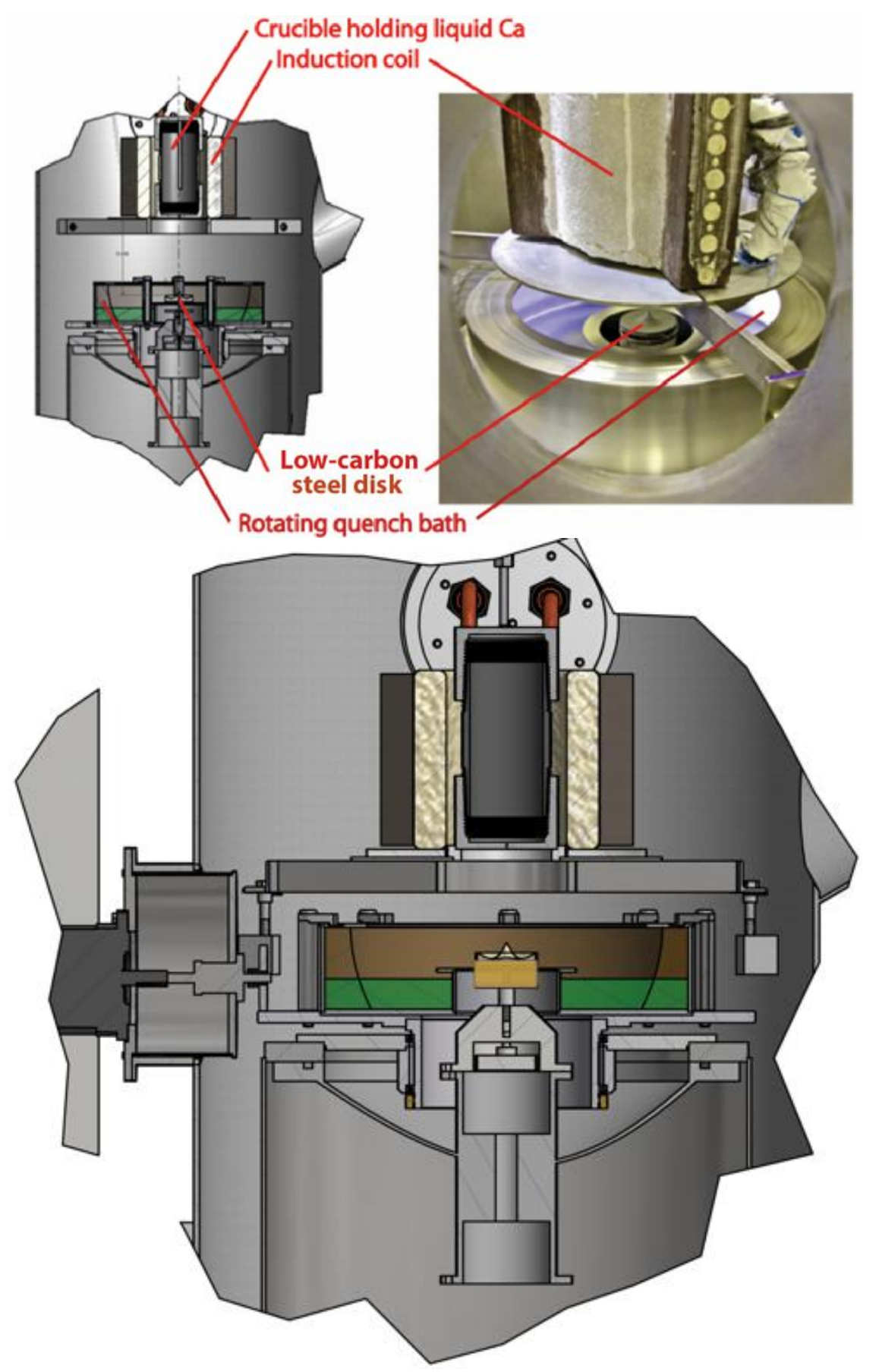

Fig. 2. Ames centrifugal atomizer for producing fine Ca metal powders: mechanical design drawing (top left), photograph of the atomizer showing induction heating coil, atomization disk, and rotating quench bath (top right). A large version of mechanical design drawing is given at bottom. 


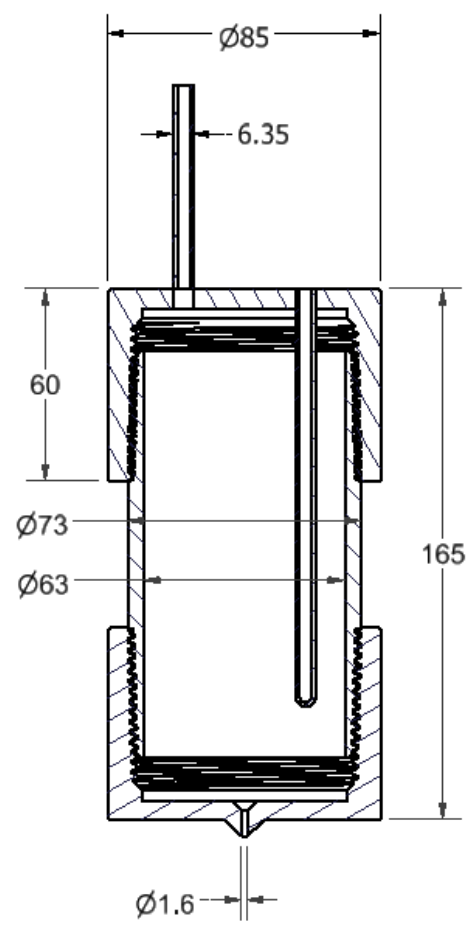

Fig. 3. Mechanical drawing of the crucible assembly. Dimensions are in $\mathrm{mm}$.

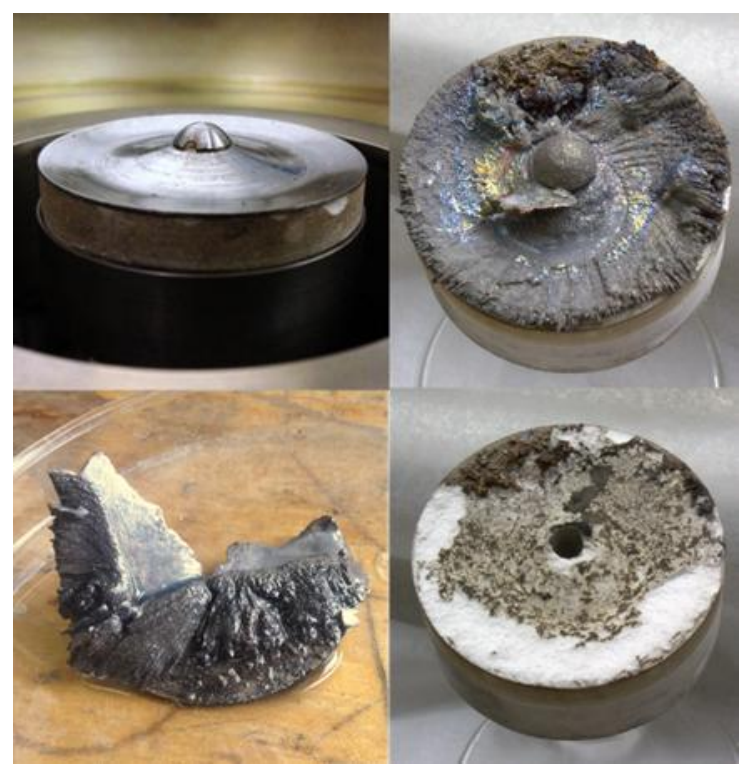

Fig. 4. The atomization disk before the experiment (upper left). The broken mild steel disk during the atomization (bottom left). The remaining solid Ca skull covered on the ceramic disk after the experiment (upper right). The ceramic disk after the skull was removed (bottom right). Disk rotation was counterclockwise. 


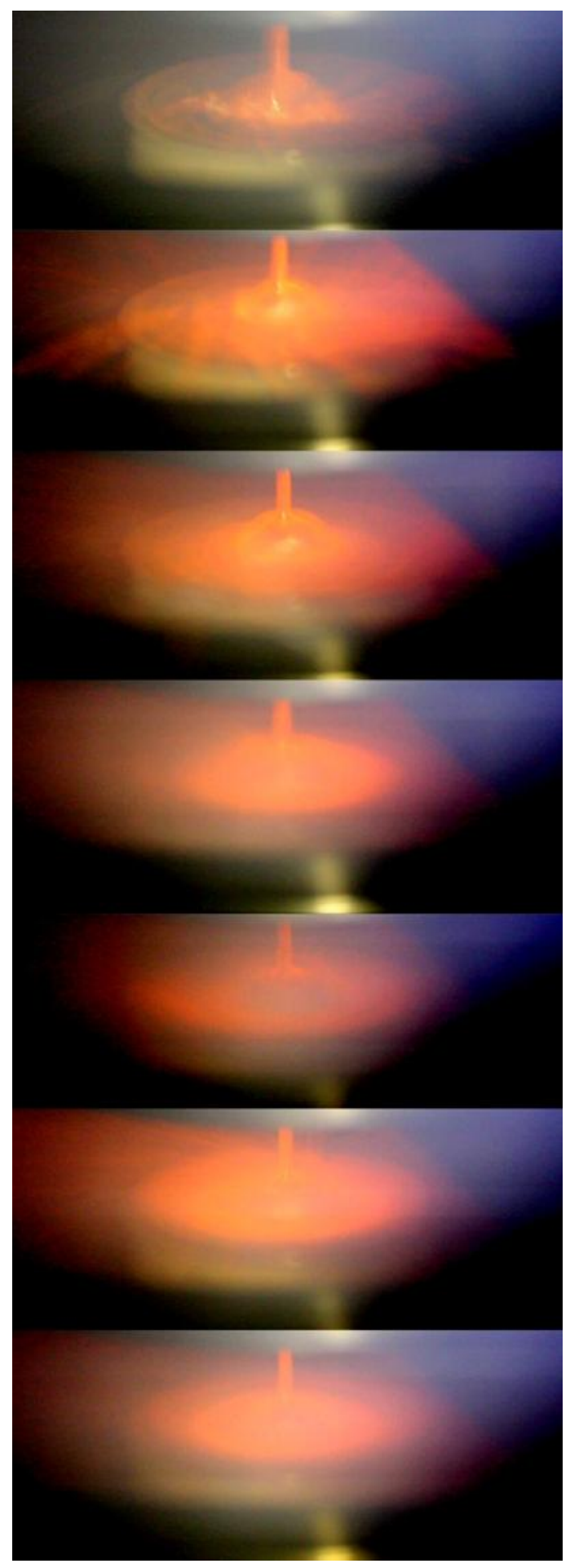

Fig. 5. Continuous photograph frames of the initiation of liquid Ca metal atomizing with time progressing from the top to the bottom under Ar atmosphere at $30 \mathrm{fps}$. 

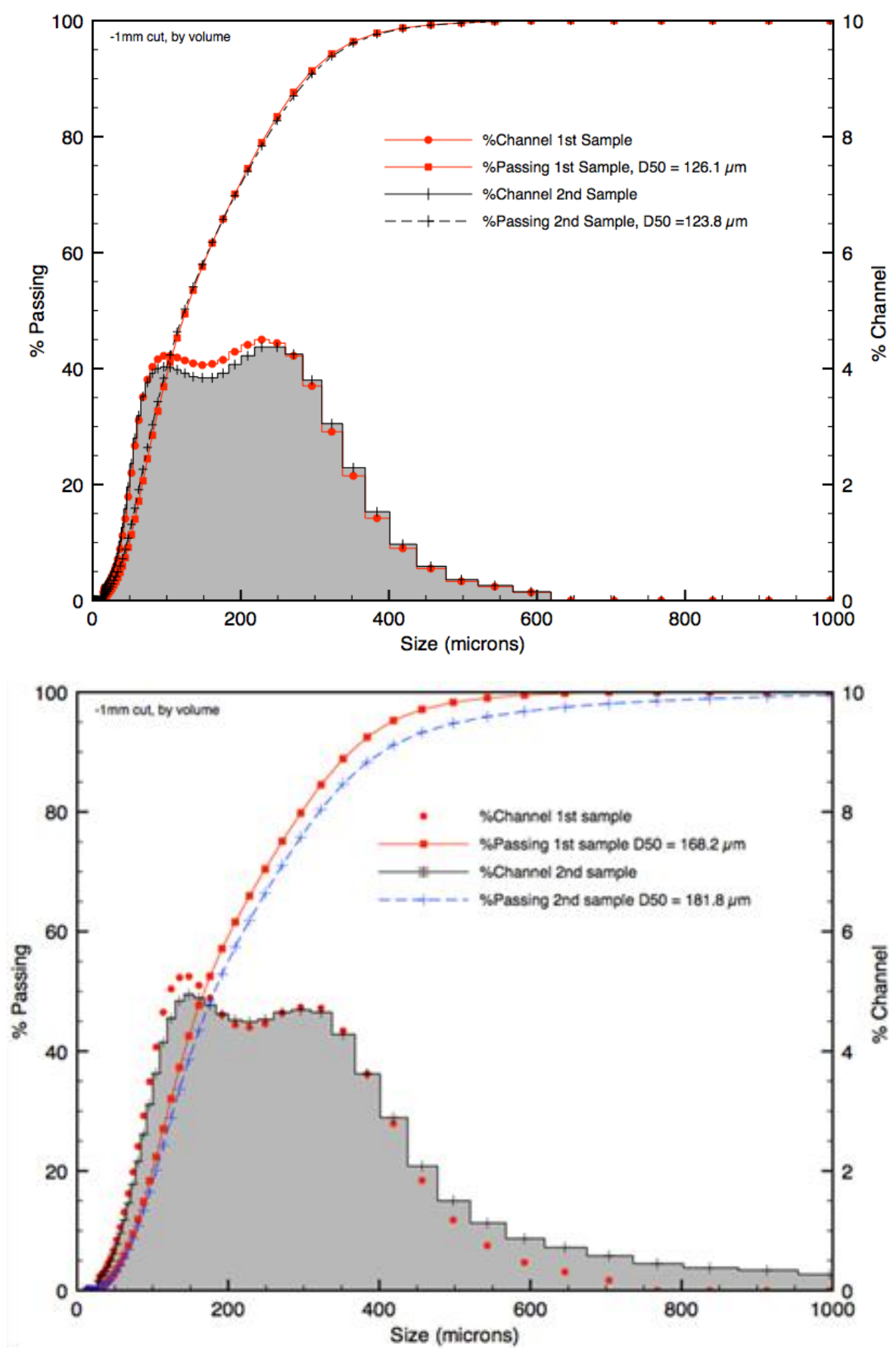

Fig. 6. The particle size distributions of 2 random, clean, atomized Ca powder samples for each of two atomization runs in Ar chamber atmosphere using the same experimental apparatus. The samples were run through a $1 \mathrm{~mm}$ screen prior to analysis. 

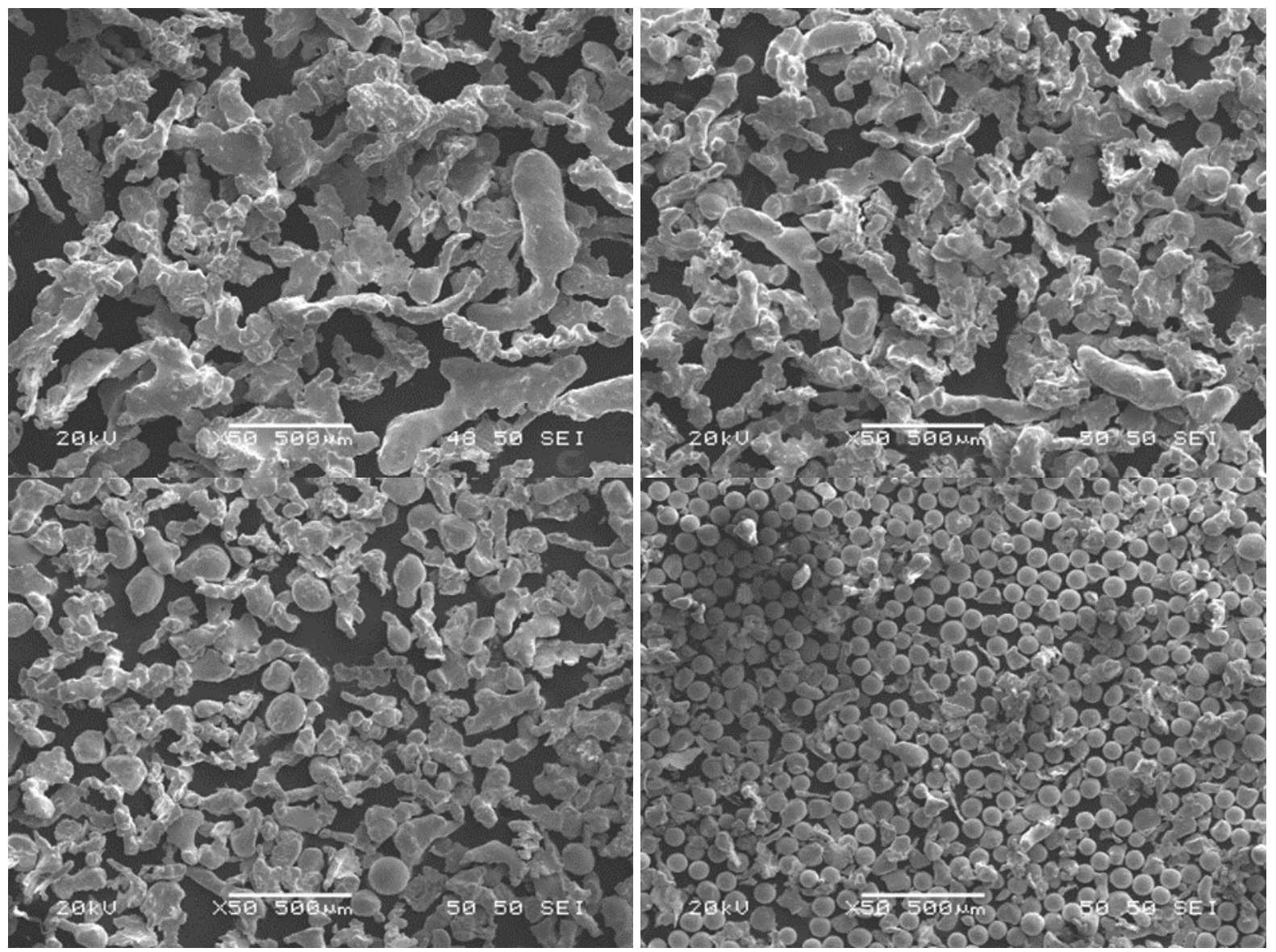

Fig. 7. Scanning electron microscope (SEM) images of centrifugally atomized Ca powders for four different size ranges: 177-250 $\mu \mathrm{m}$ (upper left); 149-177 $\mu \mathrm{m}$ (upper right); 105-149 $\mu \mathrm{m}$ (lower left); 74-105 $\mu \mathrm{m}$ (bottom right). The chamber was filled with Ar gas. 

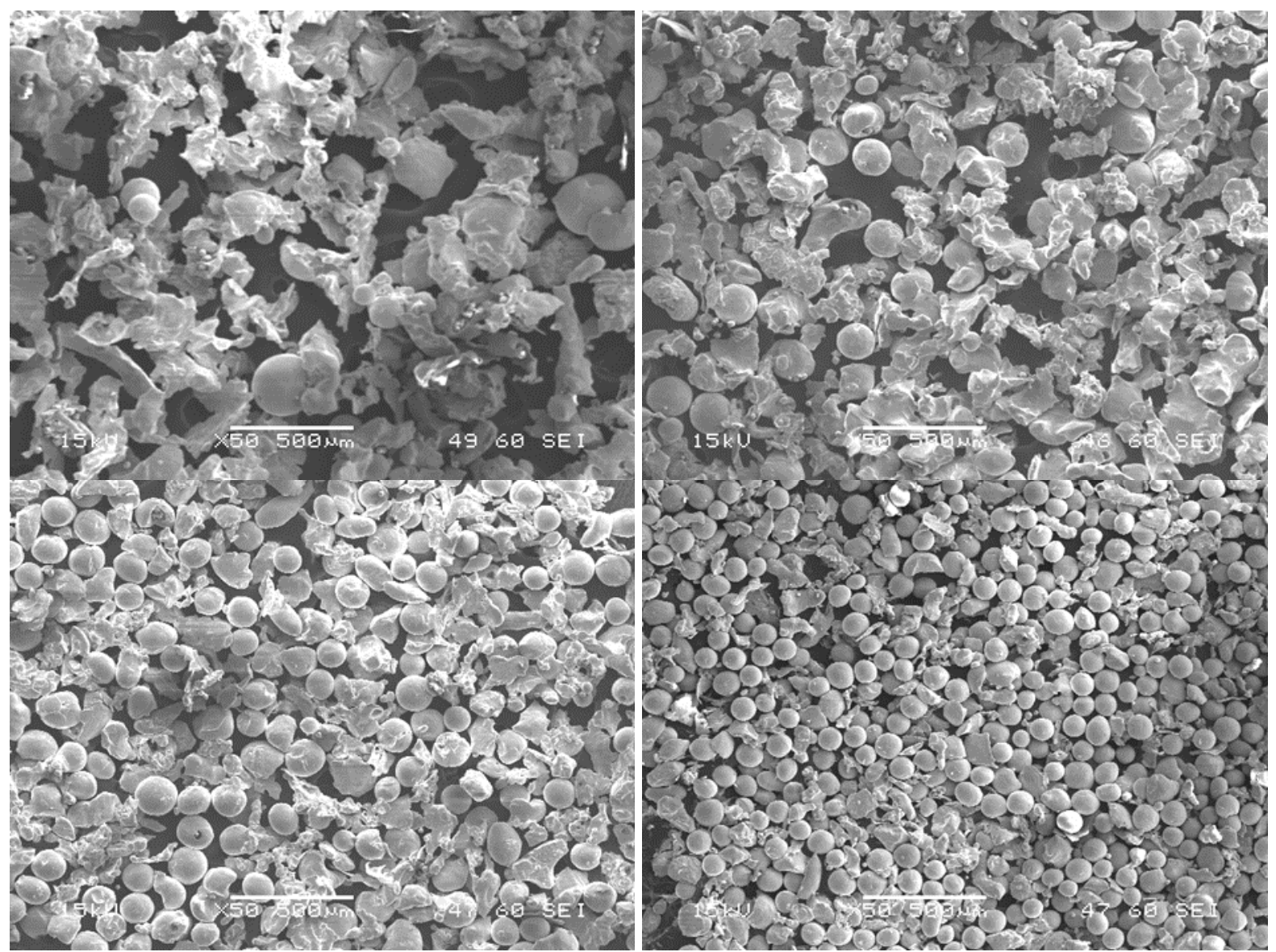

Fig. 8. Scanning electron microscope (SEM) images of centrifugally atomized Ca powders for four different size ranges: 177-250 $\mu \mathrm{m}$ (upper left); 149-177 $\mu \mathrm{m}$ (upper right); 105-149 $\mu \mathrm{m}$ (lower left); 74-105 $\mu \mathrm{m}$ (bottom right). The chamber was filled with He gas. 


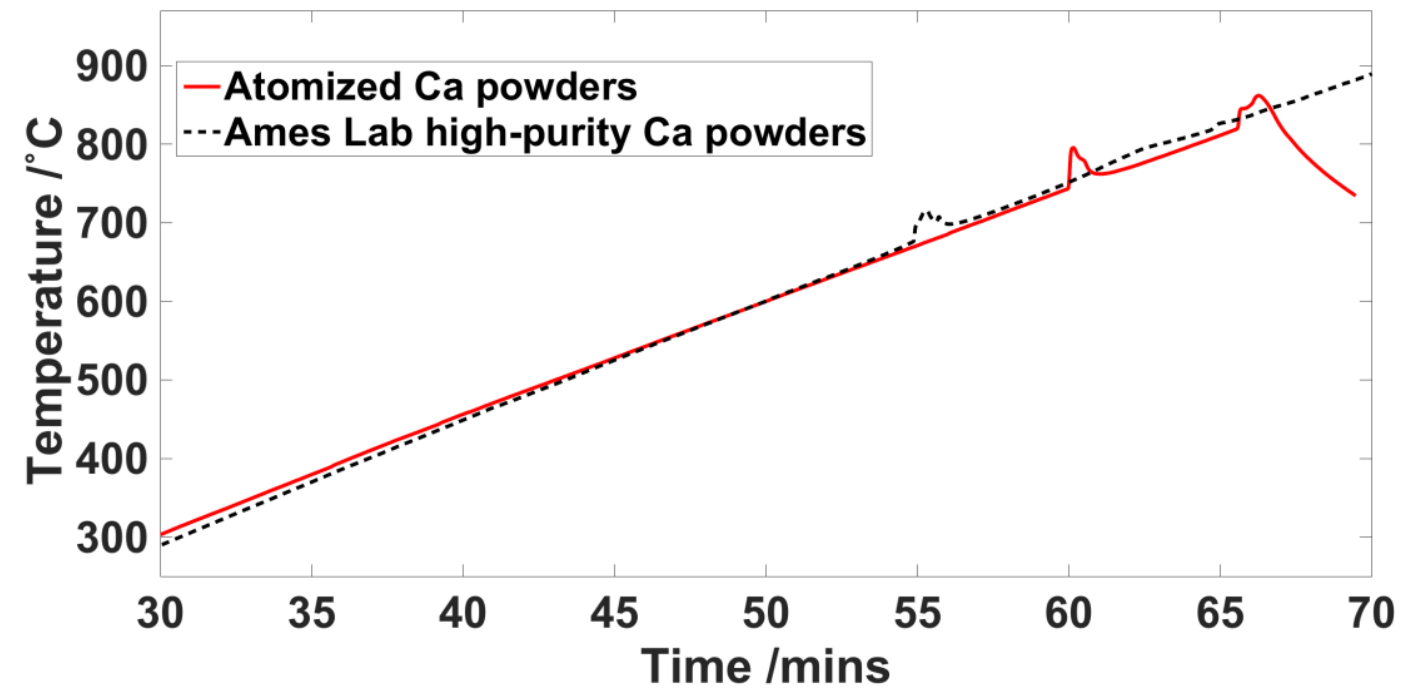

Fig. 9. Auto-ignition tests on atomized Ca powders (Mesh -70/+80) and Ames Lab high-purity Ca powder (Mesh -70/+80) in air.

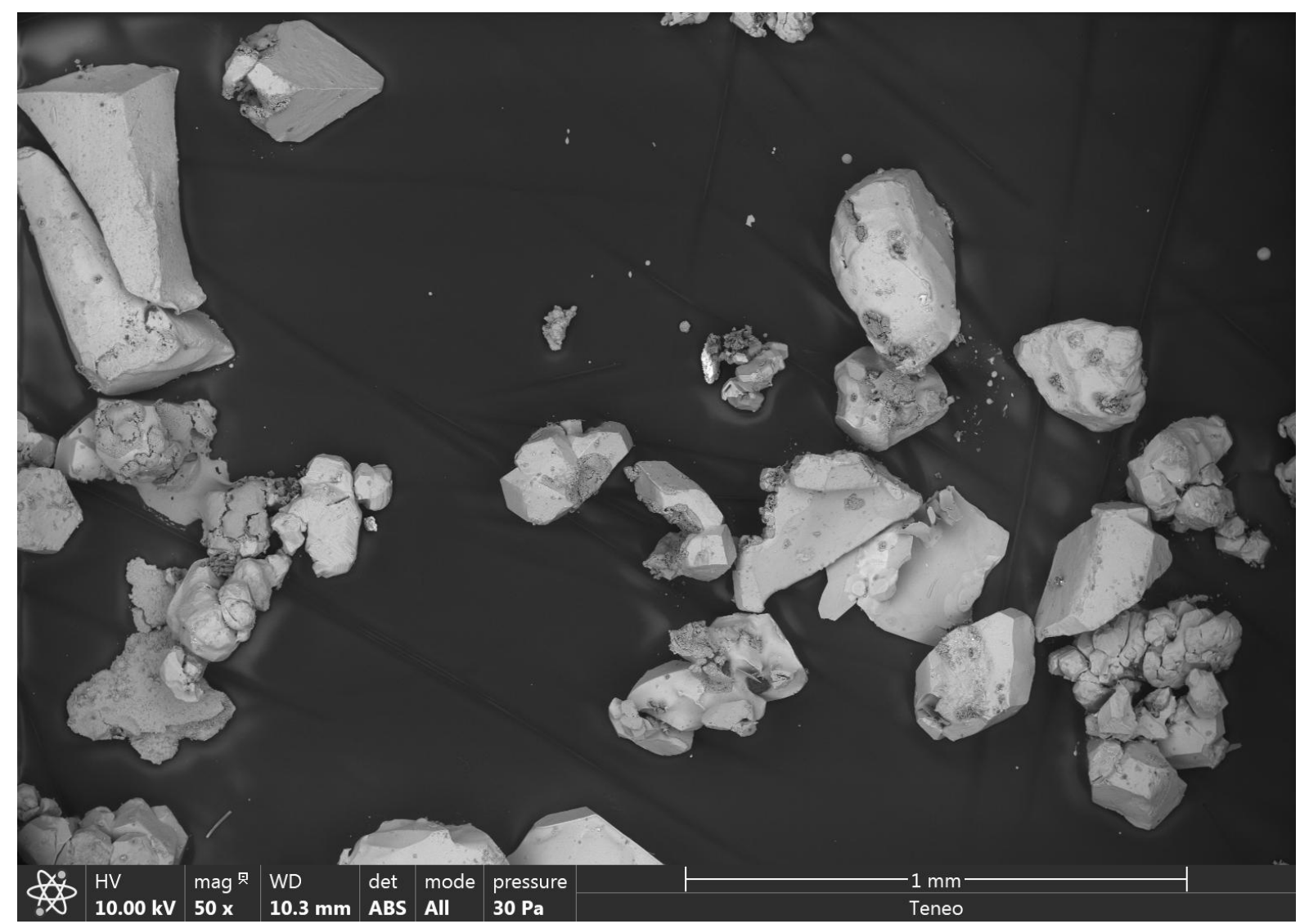

Fig. 10. Scanning Electron Microscopy (SEM) image of the particle morphology of Ames Lab high-purity sublimed Ca powders (Mesh -50/+70). 


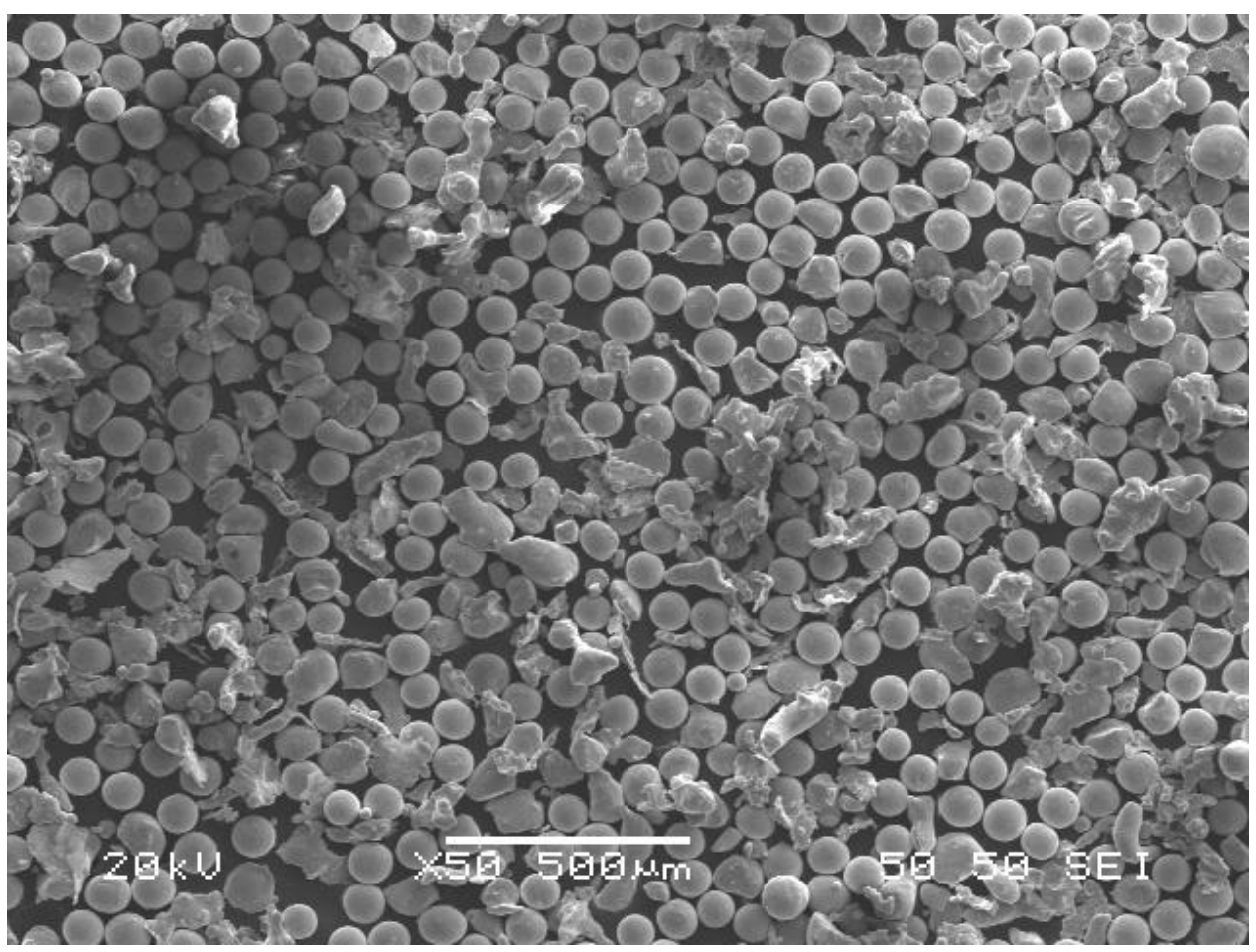

SEM picture shows fine Ca powders produced by centrifugal atomization. 